\title{
Discussion on the Importance of Scientific Literacy in Physics Discovery
}

\author{
Zhang Yao", Zhang $\mathrm{Li}^{2}$, Dang Xingju ${ }^{1}$ \\ ${ }^{1}$ College of Physics and Information Engineering, Zhaotong University, Zhaotong, Yunnan Province, \\ China \\ ${ }^{2}$ College of Foreign Language, Zhaotong University, Zhaotong, Yunnan Province, China
}

Keywords: scientific literacy, physics discovery, the history of physics, scientific knowledge, scientific thinking, scientific attitudes, scientific spirits, scientific methods

Abstract: Scientific literacy plays an important role in scientists' scientific exploration and is one of the indispensable elements of scientific research. Historical data of science and technology shows that every major scientific discovery is closely linked to scientific literacy. Physics is a key component of natural science. Therefore, this paper uses the historical data of physics as support to illustrate the importance of scientific literacy in physics discovery from five aspects.

\section{Introduction}

In the broad sense, scientific literacy includes scientific knowledge, scientific thinking, scientific attitudes, scientific spirits, and scientific methods. [1]P18The historical data of science and technology shows that scientists' extensive scientific knowledge, scientific attitudes, love of science, profound understanding of the true meaning of scientific thinking, the spirits of dedication to science, and scientific methods used in scientific research are all indispensable elements of successful scientific discoveries. Scientific literacy permeates every major scientific discovery. From another perspective, the history of physics is the development history of science and technology. [2]P95In fact, the history of physics is the history of discoveries in physics.[3]P1 This paper uses the historical data of physics as support to illustrate the importance of scientific literacy in physics discovery.

\section{Scientific knowledge lays a solid theoretical foundation for physics discovery}

To engage in physics research, physicists should have profound scientific knowledge. Scientific knowledge can broaden physicists' horizons and help them obtain scientific evidence accurately and capture the truth quickly during their exploration. Sheldon Glashow, who won the Nobel Prize in 1979, once said, "The answers to many questions in physics often go beyond the sheer scope of physics. Knowledge in multiple fields can broaden physicists' thinking." [3]P416Professor YANG Zhenning also said, "I used to study a lot of subjects in school, and my knowledge in these various subjects was further developed later through extensive contacts. This helped improve my efficiency in research." [3]P416The historical data of physics also indicates that scientific knowledge plays an important role in scientific discovery. For example, Roentgen conducted further research on the basis of Heinrich Hertz's and Philipp Lenard's research on the properties of cathode rays. On November 8, 1895, in a darkroom, in order to prevent visible rays from leaking through a Crookes tube, Roentgen used black paper to tightly wrap the tube. When the tube was connected to an induction coil and came under high voltage, Roentgen accidentally discovered weak glimmers on a fluorescent screen 1 meter away. Based on his profound knowledge, Roentgen knew that those were not cathode rays, so he carried out repeated experiments and eventually discovered a new ray, namely, the X-ray. Before Roentgen's discovery, Crookes also discovered sensitization in a photographic film near cathode ray tubes, but he mistakenly thought that the photographic film was faulty. Lenard encountered a similar phenomenon as well, but he did not conduct further research in time. Even if Lenard had conducted further research in time, it would have been difficult for him to draw the right conclusion, because he theoretically considered the $\mathrm{X}$-ray to be a cathode ray with 
infinitely large speeds. This shows that for scientists to make major discoveries in physics, they need to have profound knowledge and the correct judgement.

If physicists don't have relevant scientific knowledge, they may not be able to give scientific explanations of the phenomena that they observe during research, thus missing opportunities of physics discovery. For example, in January 1932, during their observation of beryllium radiation, the Joliot-Curie couple, misled by the erroneous thinking of Bothe, missed the opportunity to discover neutron. Later, the Joliot-Curie couple said that had they read and understood Rutherford's 1920 lecture, they would have had a correct understanding of what they observed during the experiment. [4]P260In 1932, Anderson observed the trajectory of the positive electron in his cloud chamber, and based on his profound knowledge, he concluded decisively that this was the positive electron predicted by Dirac in 1928. Prior to this discovery, the Joliot-Curie couple had also observed the trajectory of the positive electron, but once again they gave the wrong explanation, thus missing a second opportunity of major physics discovery. This tells us that scientific knowledge lays a solid theoretical foundation for physics discovery.

\section{Scientific thinking orients physics discovery in the right direction}

In the development history of science and technology, there are two levels of scientific thinking. At the first level, scientific thinking is embodied in the extraction of various special scientific knowledge and research methods into the formation of reasonable ideas and inference rules that can be used to discover and explain other phenomena or things of the same nature and to provide guidance for scientific research and social practice. At the second level, scientific thinking is embodied in scientists' achievements in scientific activities and the gradual formation of scientific awareness and scientific spirits shared by all scientists.

In 1942, American sociologist Robert K. Merton defined four institutional imperatives of the scientific ethos: universalism, communism, disinterestedness, and organized skepticism. These became a set of universal norms for scientists. For example, after Roentgen discovered the X-ray, some proposed that the X-ray should be defined as "Roentgen ray", but Roentgen insisted on calling it the "X-ray". At that time, a Berlin general electricity association wanted to buy the patent right of this new discovery at a high price, but Roentgen flatly rejected that idea and gave up the patent right of his own accord. Thanks to his contribution, the X-ray began to be widely applied in medical science, benefiting the whole world, while Roentgen himself spent his whole life in poverty. In accordance with Roentgen's will, the Nobel Prize money that he won was donated to the University of Wuerzburg as scientific research funds. Such selfless contributions by Roentgen exemplify the communism and disinterestedness articulated by Merton. Roentgen's scientific thinking encourages generations of physicists to make selfless contributions to physics. Another example is the Joliot-Curie couple's discovery of radium, a radioactive substance. As radioactivity is useful in cancer treatment, in order to alleviate cancer patients' pain, the Joliot-Curie couple refused to patent radium, and instead they disclosed the method for extracting radium. They maintained that radium belonged to all humans and that they should not make money out of this discovery. Sadly, the Joliot-Curie couple themselves could not afford even one gram of radium for their research. It was with the donations made by the American people that this couple could eventually buy radium and continue their research. Madame Curie devoted her whole beautiful life to the discovery and studies of radioactivity. Due to long-term exposure to radioactivity without protection, Madame Curie contracted leukemia and died on July 4th, 1934 in France. Becquerel discovered natural radioactivity, opening the door to nuclear physics. But due to long-term exposure to radioactivity in research, he became the first physicist to be killed by radioactive substances. It is the universalism, communism, and disinterestedness of science that have encouraged physicists to devote themselves to physics discovery, thereby advancing the development of physics.

Organized skepticism can not only help physicists fight against religious superstition, pseudo-science, and old science in scientific discovery to explore the truth but also help them distinguish between science and pseudo-science correctly, thereby providing guidance for scientific research. For example, Copernicus's idea of heliocentrism subverted Ptolemy's geocentrism, which 
had been dominant in astronomy for a long time, and this was an attack on religious theology. It changed people's view on the universe and advanced the development of physics. Galileo disproved Aristotle's wrong theory in mechanics, laying a solid foundation for Newton to complete his great work in classical mechanics. In 1915, Einstein proposed "special relativity", evolving humans' view of time and space from the mechanics of Galileo and Newton to relativity and triggering a major revolution on how humans viewed time and space. These major discoveries in physics not only advanced the development of physics itself but also changed the thinking of physicists in that particular era and beyond. New scientific thinking will lead physicists to keep exploring new fields.

\section{Scientific attitudes are a strong guarantee of physics discovery}

Physics is about the study of objective things, not just nature itself but also products generated from mutual interactions between cognitive subjects and natural objects. When studying natural objects, scientists must have scientific attitudes such as curiosity, respect for real evidence, and critical thinking. For example, in the world of particle physics, particles move in accordance with symmetry. In 1956, Chinese American physicists LI Zhengdao and YANG Zhenning, on the basis of comprehensive research and critical thinking, proposed the scientific idea of parity non-conservation in weak interactions. They also proposed that this idea should be verified by experiments, such as $§$ decay. Specifically, they said two experiments containing weak interactions should be set up as mutual mirroring, and the results of these experiments should be compared to see if they were consistent; if the results were not consistent, parity non-conservation was verified. To conduct these experiments, which were very difficult and required high precision, one should have curiosity for exploration and great fortitude. LI Zhengdao and YANG Zhenning's idea was questioned by most physicists, including WU Jianxiong, who was also of Chinese descent. However, out of respect for real evidence, WU first carried out a high-precision experiment on parity conservation, using 60Co. The result demonstrated parity non-conservation during $\beta$ decay, thereby proving the correctness of parity non-conservation in weak interactions. WU once said, in recollection of this experiment, "Although I thought the chance of parity conservation being disproved was very slim, I still wanted to do this experiment." This major scientific discovery was made possible by the strict scientific attitudes of these three physicists, YANG Zhenning, LI Zhengdao, and WU Jianxiong.

\section{Scientific spirits are the motivation and support of physics discovery}

Scientific spirits have very rich contents, including the rational spirit of truth seeking, the strict spirit of practicality, and the critical and aggressive spirit of innovation. [6]P103Many instances in physics have shown that when an old scientific theory comes into contradiction, scientists must have the rational spirit of truth seeking and the critical and aggressive spirit of innovation, as well as the courage to break away from traditional ideas that hamper scientific development, in order to come up with new thinking and new ideas, and they must also have the strict spirit of practicality in order to prove those new theories and ultimately make breakthrough discoveries. For example, Einstein's establishment of relativity, Bohr's proposal of complementarity, LI Zhengdao and YANG Zhenning's proposal of parity non-conservation in weak interactions, etc. all demonstrated physicists' spirit of breaking from traditional ideas and embracing innovation, which led to their breakthroughs in the theories of physics. Here's another case in point: Galileo, in his attempts to check and disprove Aristotle's motion theory, repeated the inclined plane experiment hundreds of times, and ultimately grasped the principle of inertia, proving that force is the cause of motion changes, and disproving Aristotle's argument of force creating motions. This physics discovery put kinetic studies on the right track. In 1820, after Oersted discovered the magnetic effects of electric currents, physicists began to carry out experiments and research on magnetic electricity generation. After repeated failures in experiments, many physicists, including those at the forefront of electromagnetism research, such as Fresnel, Ampere, and Arago, began to doubt whether magnetic power could really generate electricity. They dropped out of research on this topic. In contrast, 
Faraday, by virtue of his strict spirit of practicality and perseverance, spent a decade doing numerous experiments and eventually made a major discovery. He once wrote down a brilliant vow to "convert magnetism into electricity" in his diary, and he never questioned the possibility of this vow. [3]P286

\section{Scientific methods are the basic guarantee of physics discovery}

Good tools are prerequisite to the successful execution of a job. The development history of physics is the evolution history of scientific methodology. G.Holton, a British professor of the history of science, once said, "There is no one method for scientific discovery." Every scientist uses his or her own methods for scientific exploration, and the research methods used by physicists who have accomplished achievements in physics are often summarized into scientific methods and emulated by explorers, which then leads to more discoveries in physics. Therefore, scientific methods are the basic guarantee of physics discovery. For example, Galileo is reputed as the founder and originator of modern experimental science. His experimental and mathematical research methods creatively combined experience with rationality, qualitative studies with quantitative studies, thus profoundly influencing physics and natural science. American modern mathematician Morris Kline praised Galileo's scientific methods as the most profound and most effective thinking in scientific methodology of all time. [7]P126Newton's achievements in physics were also closely linked to his analysis-synthesis method, which emphasizes the importance of experimentation in scientific theories, meaning that the establishment of scientific principles should be based on experiments. Einstein advocated using the idea of unity of the world to intuitively observe the nature of things and using logical thinking and mathematical tools to construct theoretical systems. [7]P250His scientific method played a huge role in the establishment of the relativity theory. The scientific thinking of these great physicists has a profound impact on generations of physicists after them.

Scientific methods can help physicists improve their efficiency in scientific exploration and are the basic guarantee of physics discovery. For example, in 1931, the Joliot-Curie couple used a new method to carry out an experiment on the basis of the beryllium radiation experiment by Bothe and Becquerel. The coupled placed many substances between beryllium and measurement instruments, and to their surprise, they found that under the radiation of the beryllium rays, paraffin emitted large quantities of protons. As the experimental method they used differed from those used by other physicists, they helped accelerate the discovery of neutron. Physicist Descartes once said, "My mind is not better than others'. It's just that I'm lucky to have ways to discover methods, and with these methods, I can gradually increase my knowledge in so far as my mediocre wisdom and short life allow." [3]P450

To sum up, scientific literacy provides a strong guarantee on all aspects of physicists' scientific research and exploration and therefore plays an important role in physics discovery.

\section{Acknowledgement}

In this paper, the research was sponsored by Fund project: project of the Teaching Research Fund of Yunnan Province Education Department (2015Y486)

\section{References}

[1] LI Yanping, SHEN Xianjia, Course of the History of Physics [M], Beijing: CSPM, 2009:18

[2] SHE Haoyun, Role of Physics Education in Improving Citizens' Scientific Literacy [J], Journal of Huaihua University, 2003 (4):95

[3] CHENG Jiubiao, ZHANG Xiankui, The Art of Physics Discovery [M], Beijing: Press Journal of Ocean University of China, 2003:1; 416; 286; 450

[4] ZHONG Kouzhuang, Course of the History of Physics [M], Nanjing: Nanjing University Press, 
2015:203

[5] SHA Zhenshun, Brief Introduction to the History of Physics (Edition 2) [M], Nanjing: Nanjing University Press, 2015:203

[6] CHEN Yajuan, Brief Discussion on the Scientific, Humanistic, and Philosophical Value of the History of Science in Education [J], People's Education Press 2009:(7):103

[7] LIU Jinyu, HUANG Liwen, Brief Introduction to the Development History of Science and Technology [M], Guangzhou: Press of South China University of Technology [M], 2009:126; 